\title{
AMCoR
}

Asahikawa Medical University Repository http://amcor.asahikawa-med.ac.jp/

Biochimica et Biophysica Acta (BBA) - General Subjects (2011.12) 10 (12):1150-1159.

Molecular cloning and functional analysis of scavenger receptor zebrafish CL-P1

Mitsuko Fukuda, Katsuki Ohtani, Seong-Jae Jang, Takayuki Yoshizaki, Ken-ichiro Mori, Wataru Motomura, Itsuro Yoshida, Yasuhiko Suzuki, Yutaka Kohgo, Nobutaka Wakamiya 


\section{MOLECULAR CLONING AND FUNCTIONAL ANALYSIS OF SCAVENGER RECEPTOR ZEBRAFISH CL-P1}

Mitsuko Fukuda ${ }^{\text {a } * \text {, Katsuki Ohtani }}{ }^{\text {a } * \text {, Seong-Jae Jang }}{ }^{\text {a }}$, Takayuki Yoshizaki ${ }^{a}$, Ken-ichiro Mori ${ }^{a}$, Wataru Motomura ${ }^{a}$, Itsuro Yoshida ${ }^{a},{ }^{*}$ Yasuhiko Suzuki $^{\mathrm{b}}$, Yutaka Kohgo ${ }^{\mathrm{c}}$, Nobutaka Wakamiya ${ }^{\mathrm{a}, * *}$

Department of ${ }^{\mathrm{a}}$ Microbiology and Immunochemistry and ${ }^{\mathrm{c}}$ Internal Medicine, Asahikawa Medical University, 2-1-1-1 Midorigaoka-Higashi, Asahikawa 078-8510, Japan

${ }^{\mathrm{b}}$ Department of Global Epidemiology, Hokkaido University Research Center for Zoonosis Control, Sapporo 060-0818, Japan

*The first two authors contributed equally to this work.

Running head: Zebrafish scavenger receptor CL-P1in embryogenesis

** To whom correspondence should be addressed: Department of Microbiology and Immunochemistry, Asahikawa Medical University, 2-1-1-1 Midorigaoka-Higashi, Asahikawa 078-8510, Japan.

Tel.:+81-166-68-2393; Fax:+81-166-68-2399; E-mail address: wakamiya@asahikawa-med.ac.jp

Keywords: Collectin, Scavenger receptor, Innate immunity, Zebrafish, Embryogenesis 


\begin{abstract}
Background: Scavenger receptors are generally expressed in macrophages and vascular endothelial cells and some scavenger receptors are thought to contribute to the development of atherosclerosis.

Methods: We cloned the cDNA of a zebrafish CL-P1 (collectin placenta 1 ) and performed a knockdown study using its antisense morpholino oligonucleotides (MO).

Results: Zebrafish CL-P1 (zCL-P1) is 51\% identical to human CL-P1 in its amino acid sequence. Microbes and OxLDL bound to zCL-P1 cDNA transfected cells. zCL-P1 mRNA expression gradually increased after 6 hours post-fertilization (hpf), reached its highest level at $24 \mathrm{hpf}$, and then decreased, which is similar to the gene expression pattern of Tie-2. The knockdown of zCL-P1 led to an increase in the number of zebrafish embryos with severe morphological abnormalities such as short body lengths and defects in the dorsal aorta at $48 \mathrm{hpf}$. Simultaneous injection of both MO and synthetic zCL-P1 or zVEGF mRNA rescued the abnormal phenotype.

Conclusions: In vivo knockdown study shows that zCL-P1 is implicated in vasculogenesis and those of our in vitro study support its role as a scavenger receptor.

General Significance: These results suggest that zCL-P1 might be essential for vasculogenesis during the early embryonic phase in bone fish.
\end{abstract}




\section{Introduction}

Collectins are a family of C-type lectins harboring collagen-like sequences and carbohydrate recognition domains (CRD) [1]. They are involved in host defense through their ability to bind to carbohydrate antigens on microorganisms [2-7]. We have demonstrated recently that CL-P1 (collectin placenta 1) is a novel member of the collectin family. CL-P1 is a first membrane-type collectin which-is mainly expressed in vascular endothelial cells and can bind and phagocytose bacteria as well as yeast [8]. Furthermore, it binds to oxidized low density lipoprotein (OxLDL) as a scavenger receptor [8]. Scavenger receptors are generally expressed in macrophages and vascular smooth muscle cells, in which circulating lipids have accumulated, suggesting they might contribute to the development of atherosclerosis $[9,10]$. However, deletion studies using knockout mice indicate that the main role that scavenger receptors play in innate immunity is to eliminate invading microbes from the host body [11].

Angiogenesis and vasculogenesis are common biological processes that occur during embryogenesis and the menstrual cycle, and various genes are required for both. In the present study, we hypothesized that CL-P1 might be involved in the embryonic development of blood vessels in zebrafish since the CL-P1 gene in zebrafish has a high homology to its human and mice counterparts and it is produced in vascular endothelial cells $[8,12]$. The zebrafish embryo is excellent model for studying the genes and proteins that regulate embryonic development of blood vessels $[13,14]$. In this investigation, we carried out a knockdown study of the zebrafish CL-P1 gene and found that its deletion caused a malformation of vascular vessels and subsequent developmental defects.

\section{Materials and methods}

\subsection{Zebrafish maintenance}

Zebrafish (Danio rerio) were maintained in a laboratory breeding colony at $28.5^{\circ} \mathrm{C}$ on a $12 \mathrm{hr}$ light/12 hr dark cycle in accordance to the guidelines of the Ethics Committee of the Asahikawa Medical University. Embryos were collected from the natural mating of breeding pairs, maintained at $28^{\circ} \mathrm{C}$, and staged according to hours post-fertilization (hpf).

\subsection{Cloning of zebrafish CL-P1 cDNA}

We observed amino acid sequences of human and mouse of CL-P1 and subsequently searched the zebrafish EST database for corresponding sequences, and found several cDNA fragments with high homology. We purified the mRNA from adult zebrafish using TRIZOL LS Reagent (Invitrogen) and a NucleoTrap mRNA Mini Kit (Macherey-Nagel) and established a cDNA library using a SMART RACE cDNA Amplification Kit (BD Biosciences). To generate a part of the zebrafish CL-P1 cDNA, primers based on the human CL-P1 (hCL-P1) cDNA (5'-TGTAAAAACGACTGGGCCCTGAG-3') were used, with the cDNA library. An amplified 106-bp PCR product containing the putative CL-P1 was sequenced. In addition, the full size cDNA was obtained by reverse transcription PCR from adult zebrafish mRNA using a SMART-RACE cDNA Amplification Kit (BD Biosciences) according to the manufacturer's protocol, was subcloned into the pT7Blue T-vector (Novagen) and was sent for sequencing.

\subsection{Antibody}

Expression of the in zCL-P1 (amino acids 572-733 of zCL-P1) in E.coli was carried out as described previously [8]. The affinity-purified zCL-P1-CRDhis rabbit antisera were also prepared by previous methods [8]. The antibody titer was measured by ELISA (data not shown). The characterization of antibody was certified by western blotting using zCL-P1cDNA transfected CHO cells and hCL-P1cDNA transfected cells. Anti-Myc murine monoclonal antibody, anti-mouse-IgG-conjugated Alexa 594, anti-mouse-IgG-conjugated Alexa 488, anti-rabbit-IgG-conjugated Alexa 488, and anti-rabbit-IgG-conjugated Alexa 594 from Molecular Probes were used at the proper concentrations as indicated in the manufacturer's instructions.

\subsection{Cell culture and isolation of a transfected cell line}


Chinese hamster ovary-ldlA7 cells (CHO-ldlA7 cells) lacking functional LDL receptors was a gift of M. Krieger (MIT, USA), were maintained at $37^{\circ} \mathrm{C}$ in Ham's F-12 medium (Sigma) containing $5 \%$ fetal bovine serum (Invitrogen) [15]. A full-length zCL-P1 cDNA was amplified from our zebrafish cDNA library by PCR primers 5'-TCCCCGCGGATGAAGGACGACTTCAATGAT-3' and 5'-GAATCCAAAGTTCCAGTTTTACCGCGGGGA-3', subcloned into pcDNA3.1/Myc-His A vector (Invitrogen), sequenced, and transfected into CHO-ldlA7 cells using the polyethylenimine (PEI) based method. CHO/hCL-P1 was used as positive control cells [8]. Negative control cells were prepared to be transfected with above empty vector.

\subsection{Analysis of biochemical properties in zCL-PI}

The transient zCL-P1 transfected cells (CHO/zCL-P1) or control cells were plated at a density of $3 \times 10^{4}$ cells / $0.2 \mathrm{ml}$ in 14-mm wells of 35-mm plastic culture dishes and cultured in Ham's F-12 medium containing 5\% fetal bovine serum. Membrane immunofluorescence analysis of CL-P1 in these CHO cells was performed. CL-P1 protein was detected using $0.24 \mu \mathrm{g} / \mathrm{ml}$ anti-Myc murine monoclonal antibody and anti-mouse-IgG-conjugated Alexa 594. The microorganism binding assay was performed with Zymosan A (Saccharomyces cerevisiae), Staphylococcus aureus, and Escherichia coli BioParticles conjugated with Alexa 488 (Molecular Probes). CHO/zCL-P1 cells or control cells were incubated at $4^{\circ} \mathrm{C}$ for 30 min with $50 \mu \mathrm{g} / \mathrm{ml}$ of these three BioParticles. After binding, cells were fixed at room temperature for 30 min with 4\% paraformaldehyde in PBS and stained with anti-Myc antibody and anti-mouse-IgG-conjugated Alexa 594. In lipoprotein binding assay, cells were incubated at $4^{\circ} \mathrm{C}$ for $30 \mathrm{~min}$ with DiI-OxLDL, DiI-AcLDL, and DiI-LDL then washed with PBS, and fixed using $4 \%$ paraformaldehyde in PBS at room temperature for $20 \mathrm{~min}$. Cells were washed and incubated with anti-Myc antibody and anti-mouse-IgG-conjugated Alexa 488 [8]. After washing, all cells were treated with 1 drop of Slowfade Antifade Reagent (Molecular Probes), mounted, and sealed. A fluorescence microscopy IX 70 (Olympus) was used. The monosaccharide specificities of zCL-P1-CRDhis, hCL-P1-CRDhis, and CHO/hCL-P1 soluble extracts were analyzed using sugar blot method [7,8]. Their protein solutions were dot-blotted on the membrane and incubated with sugar BP-probes: $\alpha$-D-mannose, $\alpha$-L-fucose, $\alpha$-D-glucose, $\alpha$-D-galactose, $\beta$-D-galactose, $\beta$-N-acetyl-glucosamine, $\alpha$-N-acetyl-galactosamine, or $\beta$-N-acetyl-galactosamine. Then the membranes were incubated with streptavidine-biotinylated HRP (Chemicon International) and visualized using ECL reaction and were detected with an LAS 3000 [7].

\subsection{Real time RT-PCR analyses}

Ten zebrafish embryos each were harvested at 1-48 hpf. Total RNA was extracted using TRIZOL LS Reagent. First-strand cDNA was reverse transcribed using random hexamer primers and reverse transcriptase (TaqMan). For amplification of zCL-P1, zVEGF, zKDR, zFlt-1, zTie-2 and $\beta$-actin, we used TaqMan Gene Expression Assays and primers, and then employed an Applied Biosystems 7500 Real Time PCR System (Applied Biosystems).

\subsection{Whole-mount in situ hybridization}

Two antisense digoxygenin-labeled riboprobes of the zCL-P1 riboprobe corresponding to the CRD and a zKDR riboprobe were synthesized using a DIG RNA Labeling Kit (Roche Diagnostics). Whole-mount in situ hybridization of zebrafish embryos at $24 \mathrm{hpf}$ was performed using an In Situ Hybridization Kit (R\&D Systems) according to manufacturer's instructions. Images were observed using SZ12 stereomicroscope (Olympus).

\subsection{Immunohistochemistry}

Zebrafish embryos at $48 \mathrm{hpf}$ and larvae at 2 months post-fertilization were extracted, fixed with $4 \%$ paraformaldehyde in PBS at $4{ }^{\circ} \mathrm{C}$ for $30 \mathrm{~min}$, dehydrated, embedding in paraffin and $5 \mu \mathrm{m}$ transverse sections were prepared. Serial specimens were stained immunohistochemically or with hematoxylin-eosin [16]. After incubation with $0.01 \mathrm{M}$ citrate buffer at $95^{\circ} \mathrm{C}$ for $20 \mathrm{~min}$, sections were blocked at room 
temperature for $1 \mathrm{hr}$ and incubated with $10 \mu \mathrm{g} / \mathrm{ml}$ anti-zCL-P1 antibody or pre-immune rabbit serum as control antibody at $4^{\circ} \mathrm{C}$ overnight. The specimens were washed and incubated with anti-rabbit-IgG-conjugated Alexa 594 or anti-rabbit-IgG-conjugated Alexa 488 at room temperature for $40 \mathrm{~min}$. Fluorescent images were observed with the confocal laser-scanning microscope FV1000 (Olympus) with/without differential interference microscopic analyses.

\subsection{Preparation and injection of antisense morpholino oligonucleotides and $m R N A$ of zCL-P1 cDNA}

Antisense morpholino oligonucleotides (MO) were designed with sequences complementary to zCL-P1 cDNA starting around the initiating start codon based on the company's recommendations (Gene Tools). The MO sequences were: zCL-P1 MO 1, 5'-GTCGTCCTTCATCGTGGTCAAACTG-3'; zCL-P1 MO 2, 5'-GCACCGTTCCGTCACGAGCCAAACC-3'; $\quad$ zCL-P1 MO 5'-ACATCAATCTCATGTCAACGCGCAG-3'; zCL-P1 5-base mismatch MO 2 (MO2-5MM), 5'-GCAgCGTTgCGTgACcAGCgAAACC-3'. An inert standard MO (5'-CCTCTTACCTCAGTTACAATTTATA-3'), which has no effect on zebrafish development, was used as a control. MOs were microinjected into 1-4 cell stage embryos as described [17]. The injected embryos were then incubated at $28^{\circ} \mathrm{C}$. For preparations of $5^{\prime}$-capped mRNA, zCL-P1/pcDNA was linearized and transcribed with T7 RNA polymerase using an mMESSAGE mMACHINE In Vitro Transcription Kit (Ambion) according to the manufacturer's instructions. The synthesized mRNA and MO2 were co-injected into the yolks of embryos at 1-4 cell stages. The zCL-P1 mRNA sequence did not overlap that of $\mathrm{MO} 2$.

\subsection{Microangiography and alkaline phosphatase staining}

Fluorescein isothiocyanate-dextran (FITC-dextran) was injected into the sinus venosus of the anesthetized $48 \mathrm{hpf}$ embryos [14]. For staining zebrafish vascular endothelial cells, some embryos were picked up at $48 \mathrm{hpf}$ and fixed with $4 \%$ paraformaldehyde in PBS for $30 \mathrm{~min}$ at room temperature, then stained with nitro-blue tetrazolium chloride (NBT) and 5-bromo-4-chloro-3'-indolylphosphatase p-toluidine salt (BCIP) as described [18,19]. Visualization and photography were performed on an SZ 12 microscope (Olympus).

\subsection{Western blotting}

Western blotting was performed to identify CL-P1 protein expression in CHO/zCL-P1 and CHO/hCL-P1 and the fish embryos after fertilization. Cells and ten embryos at $48 \mathrm{hpf}$ were homogenized in 1x Tris-SDS, $\beta$-ME sample buffer, subjected to $4-20 \%$ SDS-PAGE and transferred to a polyvinylidene difluoride membrane using a semi-dry transfer apparatus. After blocking with blockace at $4^{\circ} \mathrm{C}$ overnight, the membrane was incubated with $5 \mu \mathrm{g} / \mathrm{ml}$ anti-zCL-P1 rabbit polyclonal antibody or anti-Myc monoclonal antibody for 1 hour. After washing with TBST, the membrane was incubated with HRP-conjugated anti-rabbit-IgG or anti-mouse-IgG for 1 hour. After washing with TBST, chemiluminescence was examined using the ECL reaction and detection with an LAS 3000 was carried out.

\subsection{Rescue of zCL-P1 activity by zCL-P1 or zVEGF mRNA injection to zCL-PI knockdown fish}

For the preparation of mRNA, Capped mRNA was synthesized from the zCL-P1 or zVEGF construct using mMESSAGE mMACHINE (Ambion) according to the manufacturer's instructions. The synthesized zCL-P1 mRNA or zVEGF mRNA and MO2 were simultaneously injected into embryo yolks [20]. For the control experiment, the same amount of MO2, but without the zCL-P1 or zVEGF mRNA, was used instead.

\subsection{Statistical analysis}

The results are expressed as means \pm SEM. Statistical analysis was performed by repeated measures ANOVA and a subsequent Fisher's LSD test. P $<0.05$ was considered statistically significant. 


\section{Results}

\subsection{Isolation of a zebrafish CL-P1 cDNA}

The predicted zCL-P1 ORF encodes a 740 amino acid (aa) protein that is almost similar in size to hCL-P1 (742-aa) (Fig. 1A, B) [8]. zCL-P1 contains a 39-aa putative cytoplasmic peptide, a 23-aa transmembrane domain, and a 678-aa extracellular domain harboring a coiled-coiled region, a collagen-like domain and a carbohydrate recognition domain (CRD). An endocytosis motif (Tyr-Lys-Arg-Phe) in Fig.1A is present in the intracytoplasmic domain of zCL-P1 as in hCL-P1. The hydrophobicity plot and amino acid sequence analysis showed that the domain structure of zCL-P1 is the same as that of human CL-P1 (Fig. 1B). Overall, zCL-P1 is 51\% identical to hCL-P1 and 52\% identical to mouse CL-P1 in terms of its amino acid sequence (Fig. 1C). The intraplasmic and transmembrane domains in whole domain structures in human and zebrafish CL-P1 have a high homology (79 and 83\%, respectively). The collagen domain has $62 \%$ homology to the human type and has 46 Gly-X-Y cycles compared with the 49 cycles of hCL-P1 collagen-specific tripeptides. zCL-P1 has two polycationic regions in the collagen domain which contains basic amino acids (arginine or lysine), although hCL-P1 has three regions and mouse SR-A has only one. The polycharge islands in the collagen-polymer structure form a strong binding site for negatively charged substances [21]. zCL-P1 has a C type lectin consisting of six cysteine residues as does hCL-P1, and its ligand specificity is of the galactose type (Gln-Pro-Asp) instead of the mannose and glucose types (Glu-Pro-Asn). CRD homology between humans and zebrafish is $52 \%$, less than the homology of other major domains.

\subsection{Expression and characterization of zCL-PI in $\mathrm{CHO}$ cells}

The Fig. 2A showed pcDNA3.1/Myc-His A vector using the transfection vector of zCL-P1 for CHO cells. Western blotting study in Fig. 2B using anti-myc and anti-zCL-P1 antibodies indicates that zCL-P1 has an approximate molecular mass of $120 \mathrm{kDa}$ band and $90 \mathrm{kDa}$ band in these cells as hCL-P1 [8]. This affinity-purified zCL-P1 antibody could recognize only zCL-P1 specifically. Membrane immunofluorescence analysis showed that zCL-P1 was expressed as a type II membrane protein since it was detected by an anti-C-terminal tag antibody (anti-Myc monoclonal antibody) (Fig. 2C). Microbe binding studies demonstrated yeast (Zymosan A) and S.aureus bound to CHO/zCL-P1 cells as well as CHO/hCL-P1 cells with high specificity. However, E. coli could not bind to CHO/zCL-P1 cells, which was different from those specific binding to CHO/hCL-P1 cells [8] (Fig. 2D). AcLDL as modified LDL or LDL as control failed to bind to CHO/zCL-P1 cells but only OxLDL could bind to them (Fig. 2E). Sugar blot analyses demonstrated that CHO/hCL-P1 soluble protein was specifically stained with $\alpha$-D-galactose, $\alpha-\mathrm{N}$-acetyl-galactosamine, and $\beta-\mathrm{N}$-acetyl-galactosamine. However, zCL-P1-CRD and hCL-P1-CRD was weakly stained with $\alpha$-L-fucose, $\alpha$-D-glucose, $\beta$-D-galactose, but not stained with $\alpha$-D-mannose, $\alpha$-D-galactose, or $\alpha / \beta-\mathrm{N}$-acetyl-galactosamine (Fig. 2F). The sugar binding activity between CL-P1-CRDs and CHO/hCL-P1 protein was completely different. These results indicated two possibilities that CL-P1-CRDs produced by E.coli were not well refolding or the whole extracellular molecule of CL-P1 was important for sugar binding activity.

\subsection{Gene expression of zCL-P1 in zebrafish}

A quantitative PCR examination of CL-P1, VEGF, and VEGF receptor activity showed that the transcript of zCL-P1 was present from an early embryonic stage prior to the appearance of a vascular organ (Fig. 3A). The expression peak of zCL-P1 mRNA was found at $24 \mathrm{hpf}$, after a gradual rise from the $6 \mathrm{hpf}$ stage. This expression pattern of zCL-P1 mRNA was similar to that of zTie-2 mRNA. However, the expression pattern of zVEGF mRNA was similar to mRNAs of zVEGF receptor/zKDR and /zFlt-1 $[22,23]$. Whole-mount in situ hybridization of embryos at the $24 \mathrm{hpf}$ stage using a zCL-P1 RNA probe showed that zCL-P1 mRNA was mainly localized in the dorsal aorta (DA) but slightly in the intersegmental vessels (ISV) (Fig. 3B). zKDR mRNA was observed in ISV as well as in DA (Fig. 3B). Immunohistochemical staining with/without differential interference contrast (DIC) microscopic analyses and hematoxylin-eosin staining of fish embryos at the $48 \mathrm{hpf}$ stage showed that the affinity-purified 
antibody was able to stain fish specifically (Fig. 3C) and zCL-P1 was partially localized in the dorsal aorta and dorsal vein due to with DIC (Fig. 3D). Another immunohistochemical study using adult fish at 2 months post-fertilization showed that dorsal longitudinal anastomotic vessels harboring CL-P1 form the network structure (Fig. 3E).

\subsection{Defects in blood vessel and body development detected in the zCL-P1 knockdown study}

We constructed three different CL-P1-antisense morpholino oligonucleotides (MO1, 2, and 3) whose designs were based on sequences at different sites of the 5'-untranslated region of zCL-P1 mRNA. To evaluate the potency and specificity of MO1-3, we used a five-base-mismatched (against MO2) oligonucleotide (5MM) and a control oligonucleotide (control). Using the above oligonucleotides, we then performed a zCL-P1 gene knockdown study (Fig. 4A-D, Table 1). At 48 hpf, we found various malformations such as body trunk formation collapse, shortness in length, severe trunk winding, and pericardial edema (Fig. 4B, C). We classified the strong phenotype (strong) as having severe developmental deficiencies and the weak phenotype (weak) as having features between the strong phenotype and the normal in Fig. 4B. Embryos with the strong phenotype showed less activity, and fewer heartbeats and contractions. The three MOs caused similar malformations and the rates of their respective phenotypes were MO-dose-dependent and oligonucleotide-sequence-specific (Fig. 4A, D, Table 1, 2). Western blotting analysis using whole fish embryos revealed the complete depletion of CL-P1 protein by MO2 but not by $5 \mathrm{MM}$ or the control (Fig. 4A). With alkaline phosphatase staining, we detected endogenous alkaline phosphatase in vascular endothelial cells (Fig. 4C1). The weak phenotype fish retain DA but the strong phenotype fish lose DA or ISV. By means of microangiography with an injection of FITC-dextran, we were able to visualize functional blood flow in the fish vessels and observed the abnormal blood flows in the weak and strong phenotype fish (Fig. 4C2). These two types of staining of MO-treated fish revealed vascular defects affecting the dorsal aorta and /or intersegmental vessels. MO2 at $5 \mathrm{ng}$ caused $65.7 \%$ of fish to have a dorsal aorta defect phenotype but $2.5 \mathrm{ng}$ of MO2 caused only $17.6 \%$ of fish to have this phenotype (Fig. 4D). The extent of the severe defects in vessels was MO dose-dependent.

\subsection{Co-injection of exogenous zCL-P1 or zVEGF $m R N A$ and MO2 induces recovery from vascular defects in zCL-P1 gene knockdown fish}

The synthesized zCL-P1 or zVEGF mRNA and MO2 were simultaneously co-injected into one-cell stage embryos, which caused the strongest phenotype. The 57\% of fish with a defective phenotype was also lowered to $28 \%$ after co-injection of $250 \mathrm{pg}$ zCL-P1 mRNA and this reduction was dependent on the zCL-P1 mRNA dose (Fig. 5A). The additional co-injection of 1000 pg zCL-P1 mRNA caused to clear recovery effect in the fish with strong and weak phenotypes (Fig. 5B).

The expression kinetics of zVEGF, zKDR, zFlt-1, and zTie-2 mRNAs in MO injected fish were observed at 24, 36, and $48 \mathrm{hpf}$ (Fig. 6A). We found that zVEGF mRNA was reduced and zKDR mRNA was slightly increased by MO injection at all three times. The expression of zTie- 2 mRNA was not affected by MO injection. In Fig. 6B, the $73 \%$ of fish with a total defective phenotype was lowered to $52 \%$ after co-injection of $250 \mathrm{pg}$ zVEGF mRNA and the reduction of abnormal proportion with strong phenotype was also dependent on the dose of zVEGF mRNA. However, we could not observe the complete recovery in this dose. We failed to give the higher dose as zCL-P1 mRNA because only $250 \mathrm{pg}$ zVEGF mRNA caused to the pericardial edema (Fig. 6B).

\section{Discussion}

Our work revealed in vitro characteristics of the zebrafish CL-P1 gene product as well as the manner in which CL-P1 functions in the vascular development of the zebrafish embryo. zCL-P1 has about fifity \% homology to its human and mice counterparts in amino acid sequences and its activity in vitro is also similar. In addition, zCL-P1 is expressed in vascular tissue at the mRNA and protein level at both early embryonic and adult stages. Knockdown of endogenous CL-P1 expression using three distinct MOs during embryogenesis resulted in temporal and spatial disruption of embryonic vascular development. However, the vascular defects induced by a loss of CL-P1 expression were rescued in a dose-dependent 
manner by treating these fish with additional CL-P1 mRNA.

We analyzed the time-course expression of CL-P1 mRNA in zebrafish. A previous report demonstrated that vasculogenesis in this fish starts from around $6 \mathrm{hpf}$ [24]. The expression pattern of zCL-P1 mRNA was similar to that of zTie-2 mRNA as were those of two markers of vasculogenesis, the zVEGF and zVEGF receptors of zKDR and zFlt-1 mRNAs. The time-course change in zVEGF mRNA seen in this study was quite similar to that of previous reports $[24,25]$. Furthermore, it was demonstrated by in situ hybridization and immunohistochemistry that zCL-P1 mRNA and protein expression is partially localized in the dorsal aorta in normal zebrafish embryos. These results suggest that zCL-P1 expression in zebrafish might be involved in vasculogenesis. Previous studies have reported that KDR is expressed in angioblasts at $14 \mathrm{hpf}$, and KDR-expressed angioblasts migrate medially to form dorsal aorta (so-called vascularization) [26]. We therefore hypothesized that CL-P1 might be expressed in angioblasts as is KDR, and may play a role in vasculogenesis.

To prove the biological functions of CL-P1 in zebrafish, we performed a series of experiments employing zCL-P1 gene knockdown. The gene knockdown study revealed that the loss of CL-P1 resulted in markedly abnormal phenotypes such as a defective dorsal aorta and an overly short trunk. The vascular development of zebrafish consists of vasculogenesis of the axial vessels and angiogenesis of ISV sprouting. Two reports have demonstrated that lack of the dorsal aorta in the developing stage causes a short body length in zebrafish VEGF and KDR morphants [27-29]. A small-mount injection of MO not only decreased the frequency of the DA deficiency but also increased the ISV loss. These defective phenotypes were also found in VEGF and VEGF receptor knockdown fish [29]. Pericardial edema also occurred in CL-P1 knockdown embryos as it had with VEGF. It is therefore speculated that zCL-P1 gene knockdown suppresses normal vasculogenesis, thereby inducing shortness of the body length. This speculation is supported by our results that zCL-P1 mRNA simultaneously rescued both the dorsal aorta defect and the shorter body length. We therefore conclude that CL-P1 might be involved in zebrafish vasculogenesis. Further investigation is needed to clarify the relationship between CL-P1 and VEGF, its receptors or Tie-2.

The fact that amino acid sequences of CL-P1 are highly conserved in humans and mice, strongly suggests that CL-P1 has a very important role in maintaining homeostasis and immune system [30, 31]. Furthermore, Fig. 1 shows that the intraplasmic domain is especially highly conserved, compared with the coiled-coil domain, collagen-like domain, and the CRD. There are a di-leucine motif, an acid cluster, and an endocytosis motif of YKRF sequences in its cytoplasmic domain and its 16th tyrosine residue might be phosphorylated, and could be activated as sorting signal of YTRF sequences in the endocytosis receptor [32]. CL-P1 may bind to any particular ligands during zebrafish embryogenesis so that certain signals can be transmitted downstream through the CL-P1 cytoplasmic site. Further experiments are required to demonstrate any ligands and cytoplasmic signal pathways with vasculogenesis and angiogenesis.

It has already been demonstrated that several molecules including VEGF, KDR, and NRP1 play important roles in vasculogenesis [33]. We observed the reduction of zVEGF mRNA in zebrafish embryos after MO injection in Fig .6. We detected the recovery by the additional injection of zVEGF mRNA inferior to the effect by zCL-P1 mRNA in CL-P1 knockdown fish. These results indicate that there is any relationship between zCL-P1 and zVEGF with fish vasculogenesis.

We demonstrated here that CL-P1 is involved in zebrafish vasculogenesis, and that among members of the collectin and scavenger receptor families, its role in vasculogenesis appears to be unique. Therefore, we suggest that the scavenger receptor CL-P1 would be considered as a candidate molecule with involvement in vasculogenesis. We consider this study can provide a glimpse into the functional role of the CL-P1 molecule in vivo in vertebrates. The mechanism underlying its function in the vasculature, its significance in pathological angiogenesis and vasculogenesis, and its functional conservation in mammalian development remain to be determined.

\section{Acknowledgements}

We thank Professor M. Ito and N. Sueyoshi for technical support of our zebrafish study. This work was supported by grants from Grants-in-Aid for Scientific Research $(16390161,19390227)$ and a National Project "Knowledge Cluster Initiative" (2nd Stage, "Sapporo Biocluster Bio-S") of the Japanese Ministry of Education, Culture, Sports, Science, and Technology, by a grant from Core Research for Evolution Science and Technology of the Japan Society for the Promotion of Sciences, and by the Japan Health 
Sciences Foundation KH21011. This work was also supported by grants from the Smoking Research Foundation, Hokkaido Heart Association Grants for Research, and the Takeda Science Foundation. 


\section{References}

[1] K. Drickamer. J. Biol. Chem. Two distinct classes of carbohydrate-recognition domain in animal lectins. 263 (1988) 9557-9560.

[2] Y. Kozutsumi, T. Kawasaki, I. Yamashina. Isolation and characterization of a mannan-binding protein from rabbit serum. Biochem. Biophys. Res. Commun. 95 (1980) 658-664.

[3] K. Drickamer, M.S. Dordal, L. Reynolds. Mannose-binding proteins isolated from rat liver contain carbohydrate-recognition domains linked to collagenous tails. J. Biol. Chem. 261 (1986) 6878-6887.

[4] R.T. White, D. Damm, J. Miller, K. Spratt, J. Schilling, S. Hawgood, B. Benson, B. Cordell. Isolation and characterization of the human pulmonary surfactant apoprotein gene. Nature 317 (1985) 361-363.

[5] A. Persson, D. Chang, K. Rust, M. Moxley, W. Longmore, E. Crouch. Purification and biochemical characterization CP4 (SP-D), a collagenous surfactant-associated protein. Biochemistry 28 (1989) 6361-6367.

[6] K.L. Hartshorn, E.C. Crouch, M.R. White, P. Eggleton, A.I. Tauber, D. Chang, K. Sastry. Evidence for a protective role of pulmonary surfactant protein d (SP-D) against influenza A viruses. J. Clin. Invest. 94 (1994) 311-319.

[7] K. Ohtani, Y. Suzuki, S. Eda, T. Kawai, T., Kase, H. Yamazaki, T. Shimada, H. Keshi, Y. Sakai, A. Fukuoh, T. Sakamoto, N. Wakamiya. Molecular cloning of a novel human collectin from liver (CL-L1). J. Biol. Chem. 274 (1999) 13681-13689.

[8] K. Ohtani, Y. Suzuki, S. Eda. T. Kawai, T., Kase, H. Keshi, Y. Sakai, A. Fukuoh, T. Sakamoto, H. Itabe, T. Suzutani, M. Ogasawara, I. Yoshida, N. Wakamiya. The membrane-type collectin CL-P1 is a scavenger receptor on vascular endothelial cells. J. Biol. Chem. 276 (2001) 44222-44228.

[9] A. Matsumoto, M. Naito, H. Itakura, S. Ikemoto, H. Asaoka, I. Hayakawa, H. Kanamori, H. Aburatani, F. Takaku, H. Suzuki. Human macrophage scavenger receptor: primary structure, expression, and localization in atherosclerotic lesions. Proc. Natl. Acad. Sci. U.S.A. 87 (1990) 9133-9137.

[10] A. Nakata, Y. Nakagawa, M. Nishida, S. Nozaki, J. Miyagawa, T. Nakagawa, R. Tamura, K. Matsumoto, K. Kameda-Takemura, S. Yamashita, Y. Matsuzawa. CD36, a novel receptor for oxidized low-density lipoproteins, is highly expressed on lipid-laden macrophage in human atherosclerotic aorta. Arterioscler. Thromb. Vasc. Biol. 19 (1999) 1333-1339.

[11] H. Suzuki, Y. Kurihara, M. Takeya, N. Kamada, M. Kataoka, K. Jishage, O. Ueda, H. Sakaguchi, T. Higashi, T. Suzuki, Y. Takashima, Y. Kawabe, O. Cynshi, Y. Wada, M. Honda, H. Kurihara, H. Aburatani, T. Doi, A. Matsumoto, S. Azuma, T. Noda, Y. Toyada, H. Itakura, Y. Yazaki, S. Horiuchi, K. Takahashi, J.K. Kruijt, T.J. van Berkel, U.P. Steinbrecher, S. Ishibashi, N. Maeda, S. Gordon, T. Kodama. A role for macrophage scavenger receptors in atherosclerosis and susceptibility to infection. Nature 386 (1997) 292-296.

[12] S. Koyama. Dynamics of CL-P1 in ischemia/reperfusion. Hokkaido Igaku Zasshi 37 (2005) 229-237.

[13] B.M. Weinstein, D.L. Stemple, W. Driever, M.C. Fishman. Gridlock, a localized heritable vascular patterning defect in the zebrafish. Nat. Med. 1 (1995) 1143-1147.

[14] K. Goishi, M. Klagsbrun. Vascular endothelial growth factor and its receptors in embryonic zebrafish blood vessel development. Curr. Top Dev. Biol. 62 (2004) 127-152.

[15] D.M. Kingsley, M. Krieger. Receptor-mediated endocytosis of low density lipoprotein: somatic cell mutants define multiple genes required for expression of surface-receptor activity. Proc. Natl. Acad. Sci. U. S. A. 81 (1984) 5454-5458.

[16] M. Westerfield. The Zebrafish Book: a Guide for the Laboratory Use of Zebrafish (Brachydanio rerio), University of Oregon Press, Eugene, OR (1989).

[17] A. Nasevicius, S.C. Ekker. Effective targeted gene 'knockdown' in zebrafish. Nat. Genet. 26 (2000) 216-220.

[18] G.N. Serbedzija, E. Flynn, C.E. Willet. Zebrafish angiogenesis: A new model for drug screening. Angiogenesis 3 (1999) 353-359.

[19] J.N. Chen, P. Haffter, J. Odenthal, E. Vogelsang, M. Brand, F.J. van Eeden, M. Furutani-Seiki, M. Granato, M. Hammerschmidt, C.P. Heisenberg, Y.J. Jiang, D.A. Kane, R.N. Kelsh, M.C. Mullins, C. Nusslein-Volhard. Mutations affecting the cardiovascular system and other internal organs in zebrafish. Development 123 (1996) 293-302. 
[20] Y. Yoshimura, M. Tani, N. Okino, H. Iida, M. Ito. Molecular cloning and functional analysis of zebrafish neutral ceramidase. J. Biol. Chem. 279 (2004) 44012-44022.

[21] T. Doi, K. Higashino, Y. Kurihara, Y. Wada, T. Miyazaki, H. Nakamura, S. Uesugi, T. Imanishi, Y. Kawabe, H. Itakura. Charged collagen structure mediates the recognition of negatively charged macromolecules by macrophage scavenger receptors. J. Biol. Chem. 268 (1993) 2126-2133.

[22] F. Shalaby, J. Rossant, T.P. Yamaguchi, M. Gertsenstein, X.F. Wu, M.L. Breitman, A.C. Schuh. Failure of blood-island formation and vasculogenesis in Flk-1-deficient mice. Nature 376 (1995) 62-66.

[23] P. Carmeliet, V. Ferreira, G. Breser, S. Pollefeyt, L. Kieckens, M. Gertsenstein, M. Fahrig, A. Vandenhoeck, K. Harpal, C. Eberhardt, C. Declercq, J. Pawling, L. Moons, D. Collen, W. Risau, A. Nagy. Abnormal blood vessel development and lethality in embryos lacking a single VEGF allele. Nature 380 (1996) 435-439.

[24] W. Liao, B.W. Bisgrove, H. Sawyer, B. Hug, B. Bell, K. Peters, D.J. Grunwald, D.Y. Stainer. The zebrafish gene cloche acts upstream of a flk-1 homologue to regulate endothelial cell differentiation. Development 124 (1997) 381-389.

[25] D. Liang, J.R. Chang, A.J. Chin, A. Smith, C. Kelly, E.S. Weinberg, R. Ge. The role of vascular endothelial growth factor (VEGF) in vasculogenesis, angiogenesis, and hematopoiesis in zebrafish development. Mech. Dev. 108 (2001) 29-43.

[26] D. Liang, X. Xu, A.J. Chin, N.V. Balasubramaniyan, M.A.L. Teo, T.J. Lam, E.S. Weinsberg, R. Ge. Cloning and characterization of vascular endothelial growth factor (VEGF) from zebrafish, Danio rerio. Biochimica et Biophysica Acta 1397 (1998) 14-20.

[27] B. Fouquet, B.M. Weinstein, F.C. Serluca, M.C. Fishman. Vessel patterning in the embryo of the zebrafish: guidance by notochord. Devel. Biol. 183 (1997) 37-48.

[28] A. Nasevicius, J. Larson, S.C. Ekker. Distinct requirements for zebrafish angiogenesis revealed by a VEGF-A morphant. Yeast 17 (2000) 294-301.

[29] H. Habeck, J. Odenthal, B. Walderich, H.M. Maischein, S. Schulte-Merker; Tubingen 2000 screen consortium. Analysis of a zebrafish VEGF receptor mutant reveals specific disruption of angiogenesis. Curr. Biol. 12 (2002) 1405-1412.

[30] S. Jang, K. Ohtani, A. Fukuoh, T. Yoshizaki, M. Fukuda, W. Motomura, K. Mori, J. Fukuzawa, N. Kitamoto, I. Yoshida, Y. Suzuki, N. Wakamiya. Scavenger receptor collectin placenta 1 (CL-P1) predominantly mediates zymosan phagocytosis by human vascular endothelial cells. J. Biol. Chem. 284 (2009) 3956-3965.

[31] P.M. Murphy. Molecular mimicry and the generation of host defense protein diversity. Cell 72(6) (1993) 823-826.

[32] T. Huang, H. Deng, A.W. Wolkoff, R.J. Stockert. Phosphorylation-dependent interaction of the asialoglycoprotein receptor with molecular chaperones. J. Biol. Chem. 277(40) (2002) 37798-37803.

[33] M. Klagsbrum, S. Takashima, R. Mamluk. The role of neuropilin in vascular and tumor biology. Adv. Exp. Med. Biol. 515 (2002) 33-48. 


\section{FIGURE LEGENDS}

Fig. 1. Deduced amino acid sequences and cDNA sequence, of zebrafish CL-P1 (A). The nucleotide sequence is numbered from 5' to 3'. The hydrophobicity plot and amino acid sequences analysis showed that the domain structure of zCL-P1 is the same as that of human CL-P1 (A, B). A comparison of homology between human, mouse and zebrafish CL-P1 amino acids is shown in Figure 1C.

Fig. 2. Expression and characterization of zCL-P1. The zCL-P1 ORF was subcloned into pcDNA3.1/Myc-His (+) vector, followed by transfection into $\mathrm{CHO}$ cells (A). CHO/zCL-P1, $\mathrm{CHO} / \mathrm{hCL}-\mathrm{P} 1$, and control cells were analyzed by Western blotting analyses using an anti-Myc monoclonal antibody and anti-zCL-P1 rabbit antibody (B). Membrane immunofluorescence analysis of CL-P1 in CHO/zCL-P1 cells (zCL-P1) or control cells (vector), using anti-Myc antibody and anti-mouse-IgG-conjugated Alexa 594 (C). The binding of microorganisms in CHO/zCL-P1 cells (zCL-P1), CHO/hCL-P1 cells (hCL-P1), or vector transfected cells (vector) was assayed with Zymosan A (Saccharomyces cerevisiae) BioParticles, Staphylococcus aureus BioParticles, and Escherichia coli BioParticles conjugated with Alexa 488 (Molecular Probes). After binding, cells were stained with anti-Myc antibody and anti-mouse-IgG-conjugated Alexa 594 (D). CHO/zCL-P1 cells (zCL-P1) and vector transfected cells (vector) were incubated with DiI-OxLDL, DiI-AcLDL, and DiI-LDL and incubated with anti-Myc antibody. After $30 \mathrm{~min}$ incubation, cells were incubated with anti-mouse-IgG-conjugated Alexa 488 (E). Comparison of the binding of several sugar-biotin probes to zCL-P1CRDhis (zCL-P1CRD), hCL-P1CRDhis (hCL-P1CRD), and CHO/hCL-P1 cells extracts (hCL-P1) (F).

Fig. 3. CL-P1 mRNA and protein expression in zebrafish embryos. The time-course sequence of CL-P1, KDR, VEGF, Flt-1, and Tie-2 mRNA expression in zebrafish (A). Whole-mount in situ hybridization using zCL-P1 or zKDR riboprobe was performed in zebrafish embryos at the 24 hpf stage (B). Each photo on the right shows a high power view of the area in the corresponding dorsal aorta. The arrows or arrowhead indicate the dorsal aorta or the intersegmental vessels, respectively. Cross-sections of $48 \mathrm{hpf}$ stage embryos were stained with H\&E, anti-CL-P1 antibody, or with/without DIC (C, D).The arrow or arrowhead indicates the dorsal aorta or the dorsal vein, respectively (D). The cutting position, H\&E staining (low power view), and FITC staining of CL-P1 (high power view) in the transverse section of a two-month-old zebrafish (E). The right photo shows a high power view of the area in the corresponding network vessels in the center photo. Arrowhead indicates the dorsal blood vessel.

Fig. 4. Gene knockdown study using MOs. MOs were microinjected into embryos with various concentrations of zCL-P1 MO2, 5MM or the control (A). Western blot analysis was carried out in $48 \mathrm{hpf}$ zebrafish embryos that had been injected with the standard oligonucleotide, 5MM or MO2 (A). Vascular formation was evaluated by alkaline phosphatase (ALP) staining (C1). Microangiography was performed using fluorescein isothiocyanate-dextran (FITC-dextran) (C2). Unstained zebrafish embryos at $48 \mathrm{hpf}$ (B). Embryos at $48 \mathrm{hpf}$ that had received the standard oligonucleotide or MO2 (B, C). The terms control, severe, and weak indicate the level of abnormality in the gross appearance of embryos. Each (B, C2) shows a high power view of the corresponding upper photograph. DA indicates dorsal aorta (arrow) and ISV represents intersegmental vessels (arrowhead) $(\mathrm{C} 1,2, \mathrm{D})$. The vascular defects of embryos injected with 2.5 or $5 \mathrm{ng}$ of $\mathrm{MO} 2(\mathrm{D})$.

Fig. 5. Effect of the synthesized zCL-P1 mRNA on the abnormal phenotype frequency induced by MO2. The synthesized zCL-P1 mRNA and MO2 were simultaneously injected into embryos and the phenotype was evaluated at $48 \mathrm{hpf}$. Each bar represents the means \pm SEM of $111-139$ zebrafish embryos. *p $<0.01$ and **p< 0.05 when compared with MO2 (5 ng) alone (A). The additional co-injection of 100 and 1000 pg zCL-P1 mRNA caused to clear recovery effect on the level (normal, weak, strong) of abnormality in the gross appearance of embryos (B).

Fig. 6. The expression and role of zVEGF mRNA in the abnormal phenotype induced by MO2. The expression kinetics of zCL-P1, zKDR, zVEGF, zFlt-1, and zTie-2 mRNA in MO2, 5MM MO2 injected zebrafish, or control zebrafish at 24, 36, 48 hpf (A). The co-injection of 10, 100, and $250 \mathrm{pg}$ zVEGF 
mRNA caused to some recovery effect on the level of abnormality (B). 
A

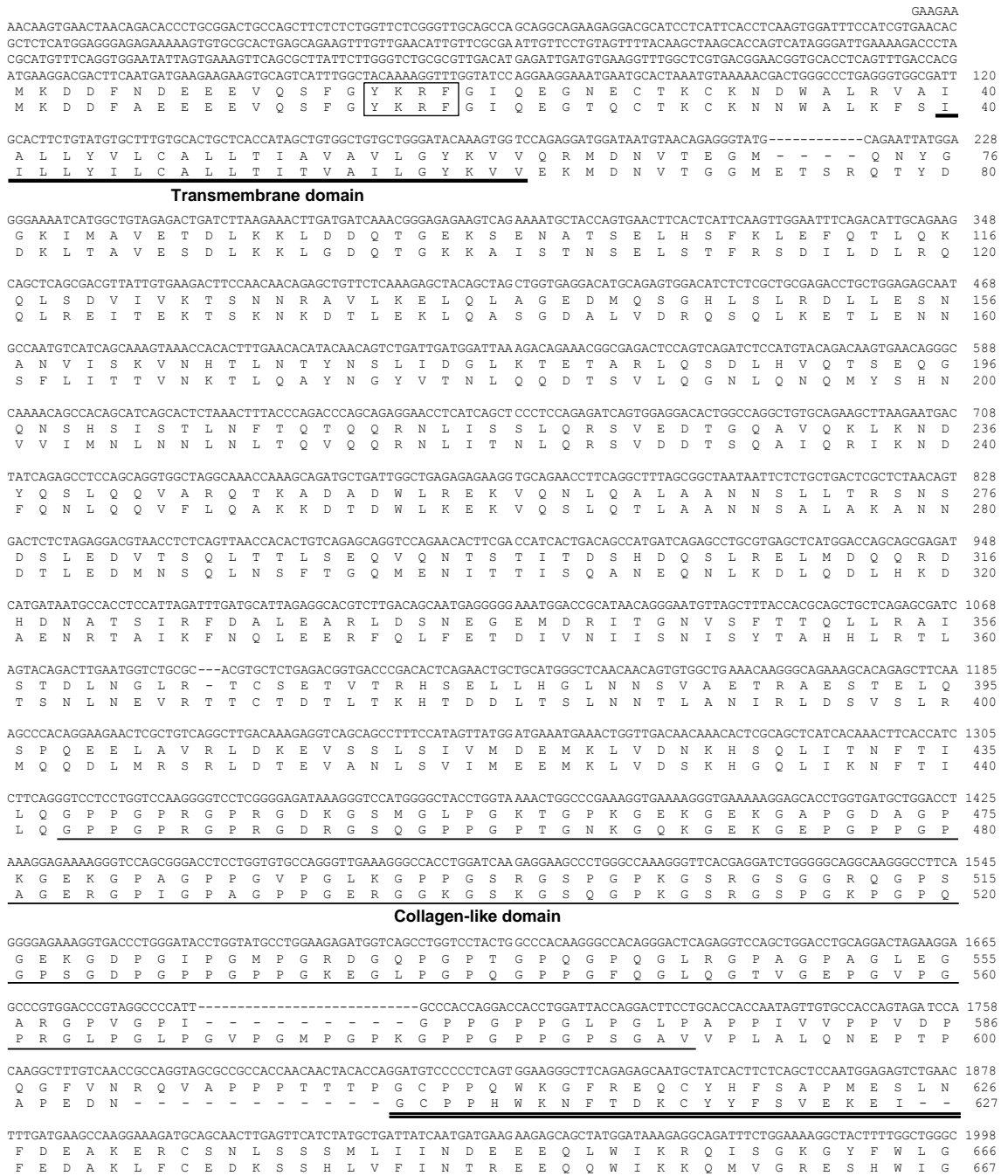
Carbohydrate recognition domain

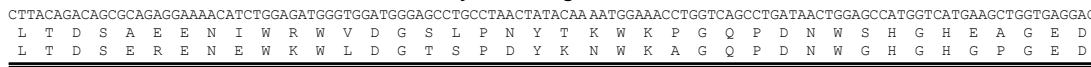

B
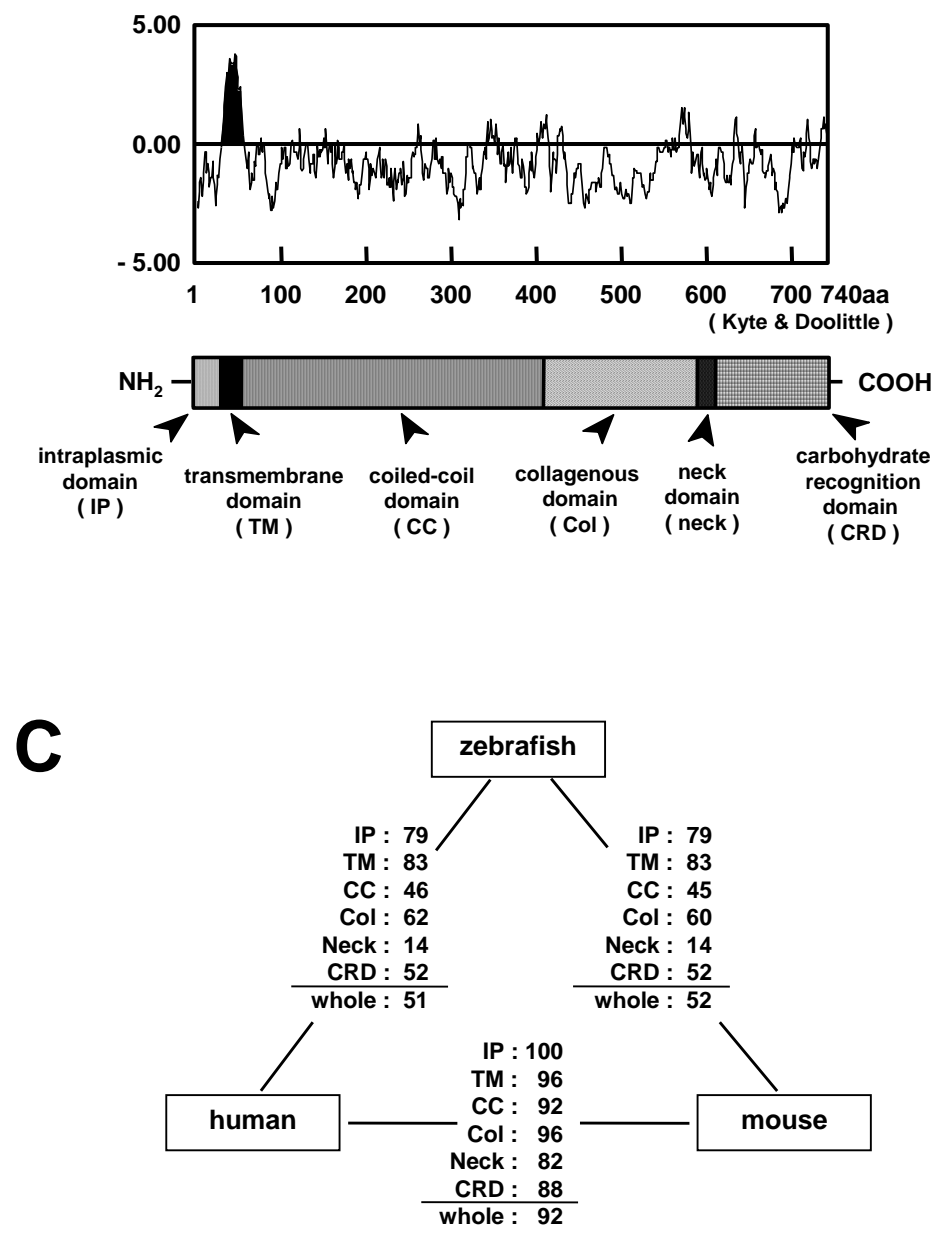
Fig. 2

A

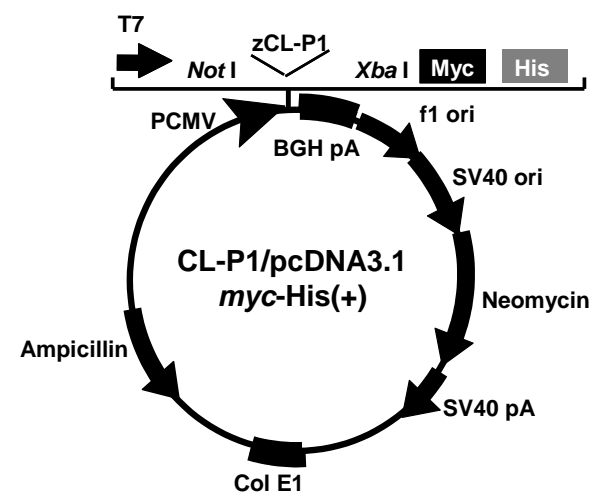

B

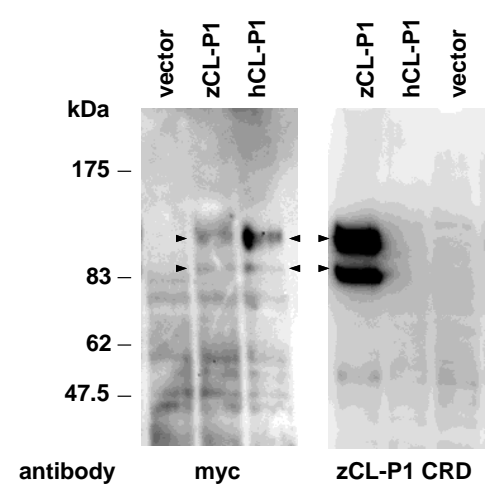

C

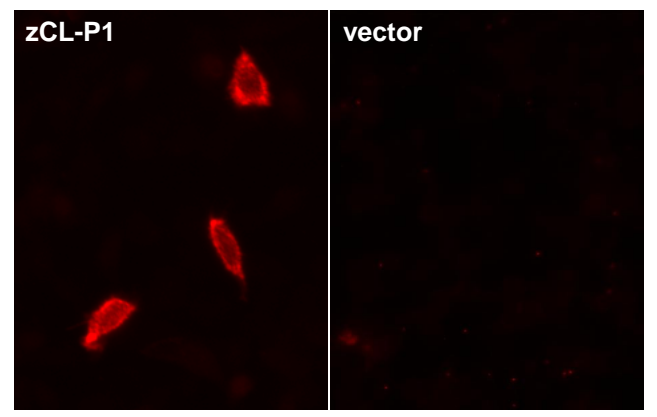

D

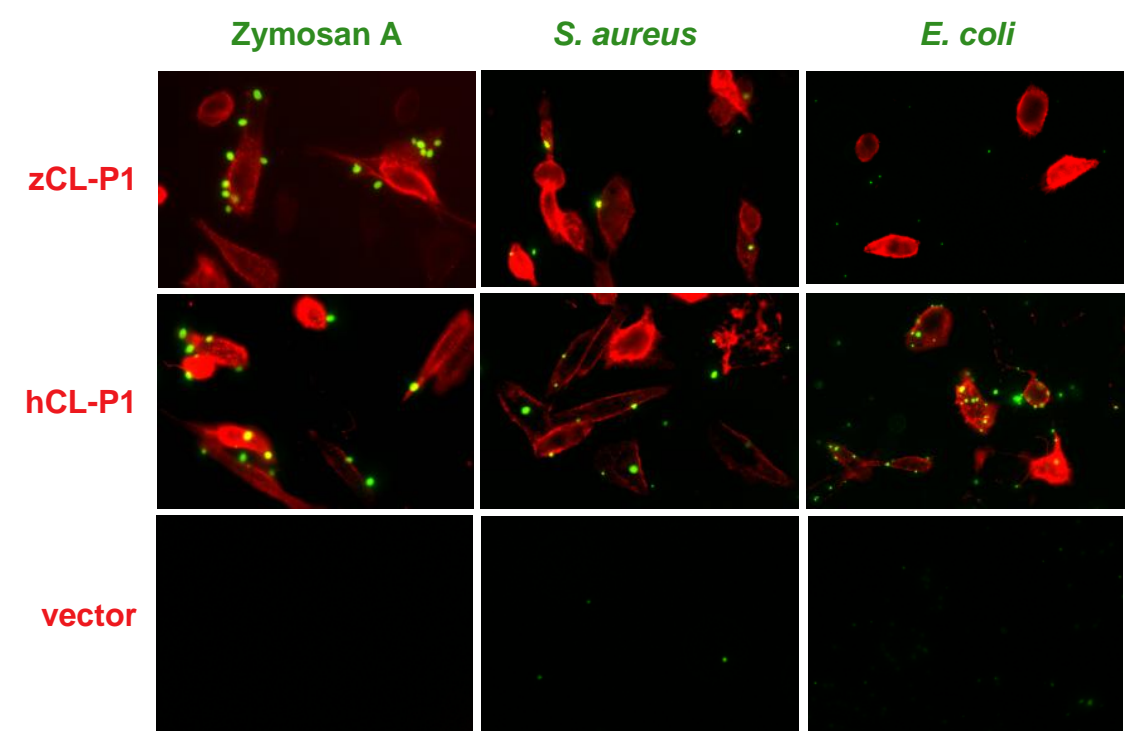

E

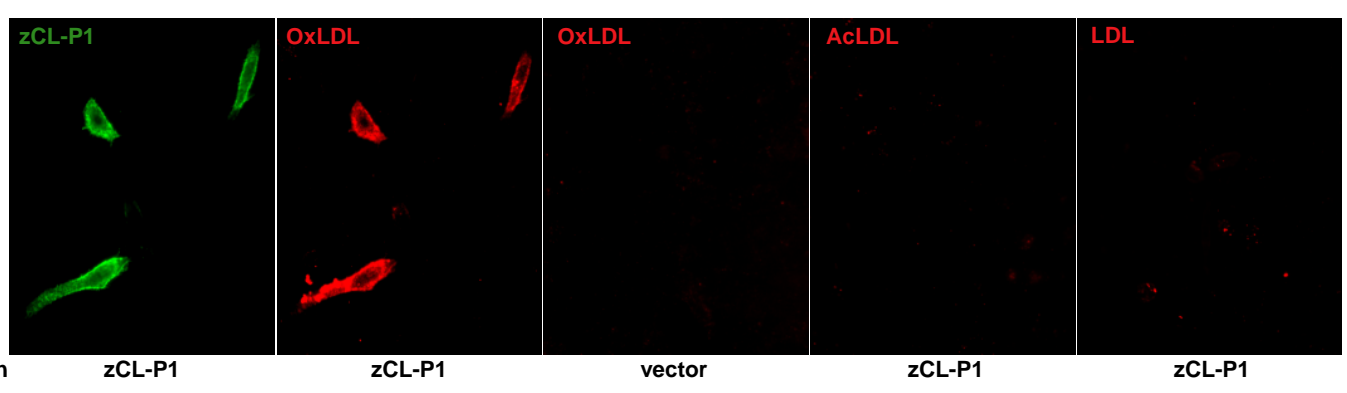

F

zCL-P1 CRD hCL-P1 CRD
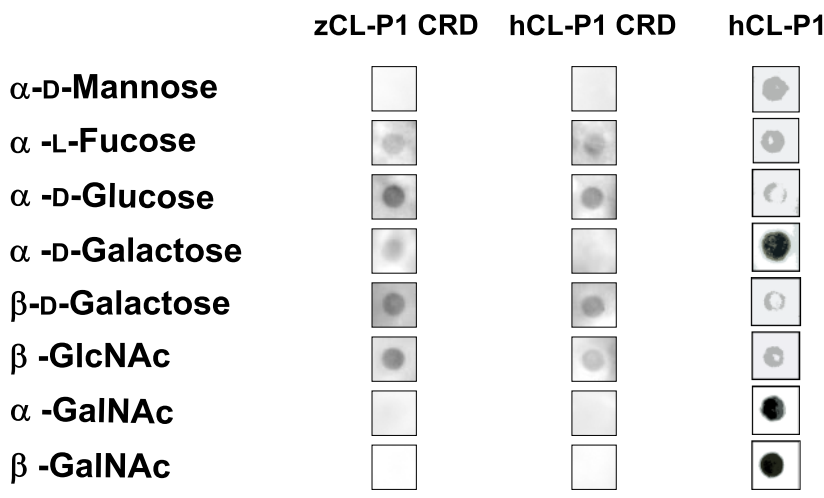
Fig. 3
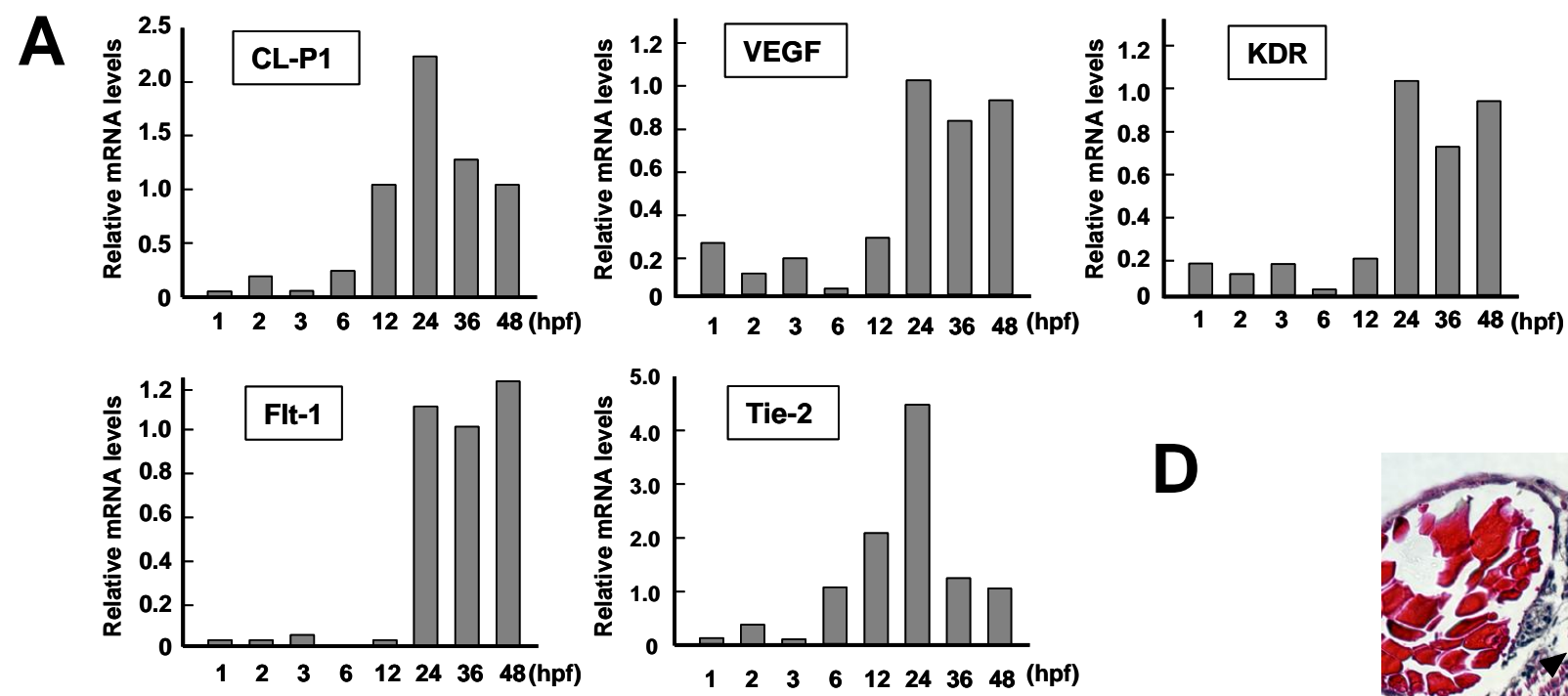

B
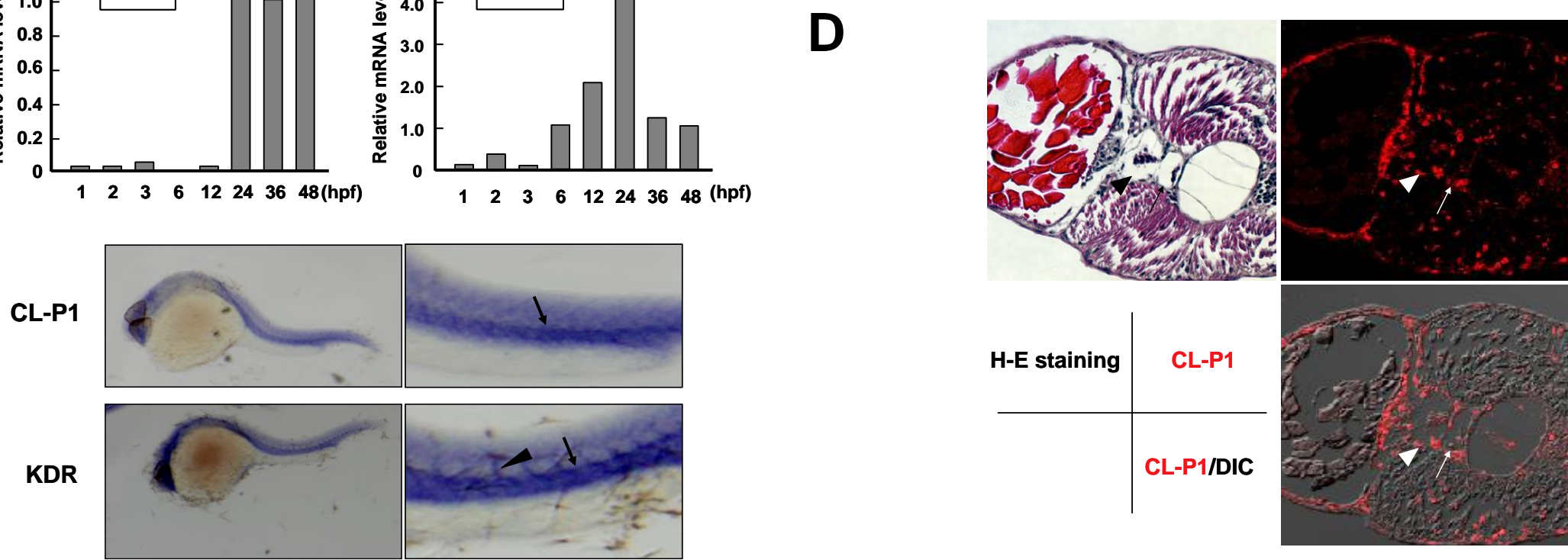

C

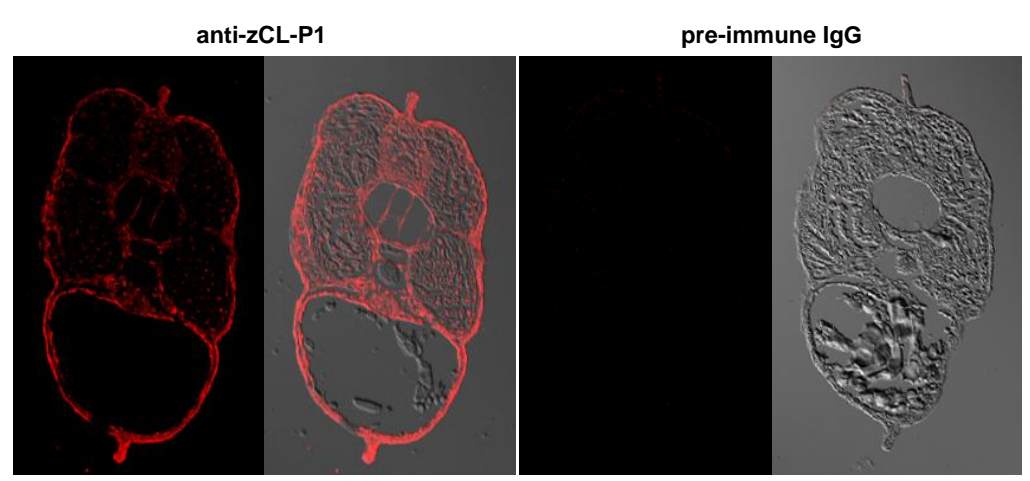

E

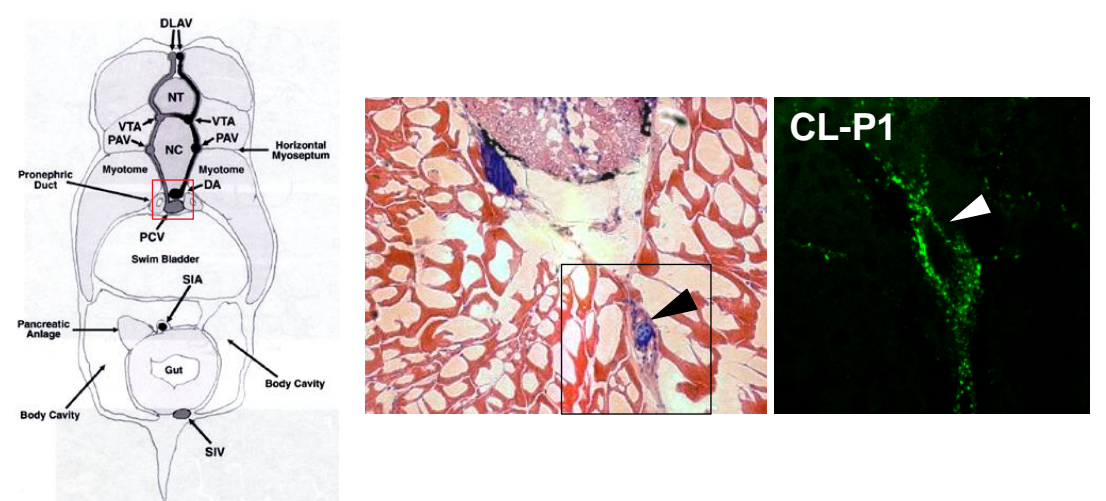


Fig. 4

A

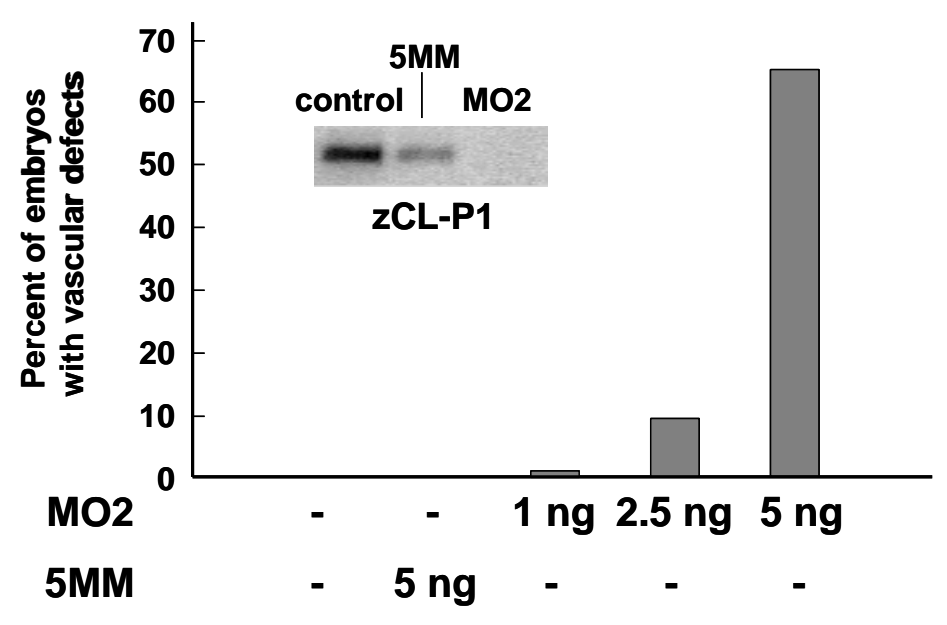

B
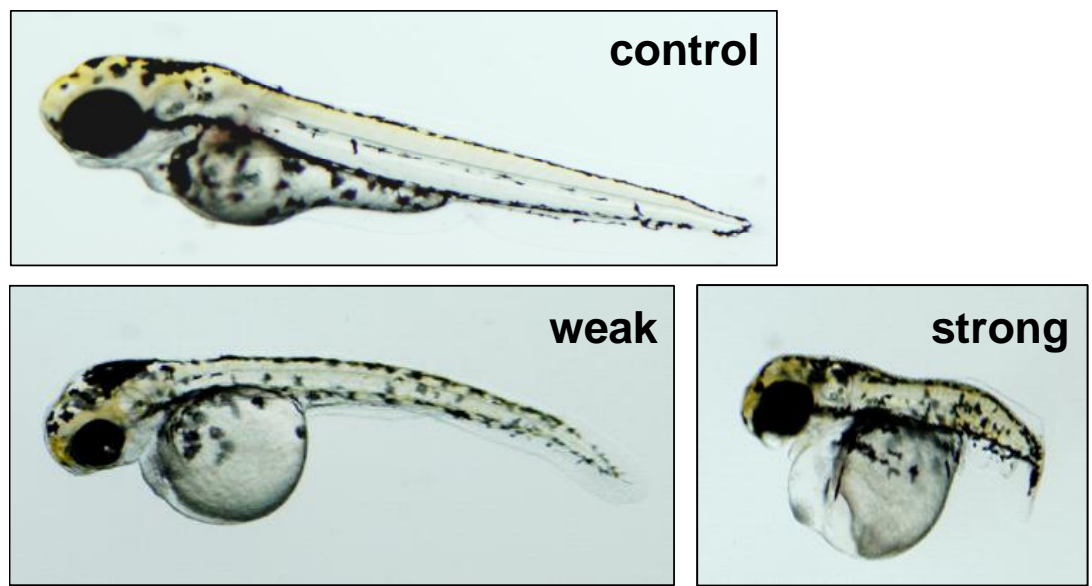
control $5 \mathrm{ng} \quad-\quad-\quad-\quad$ -

C1
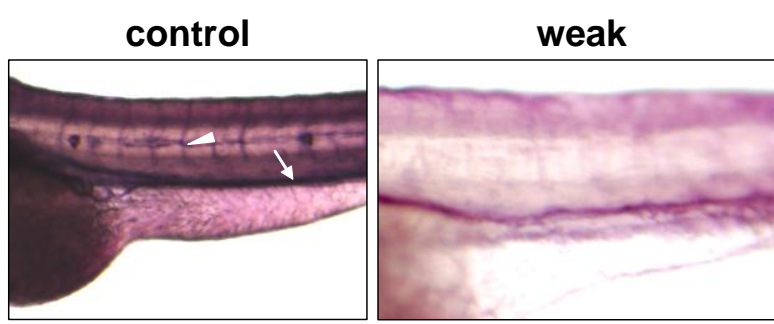

DA ISV

C2

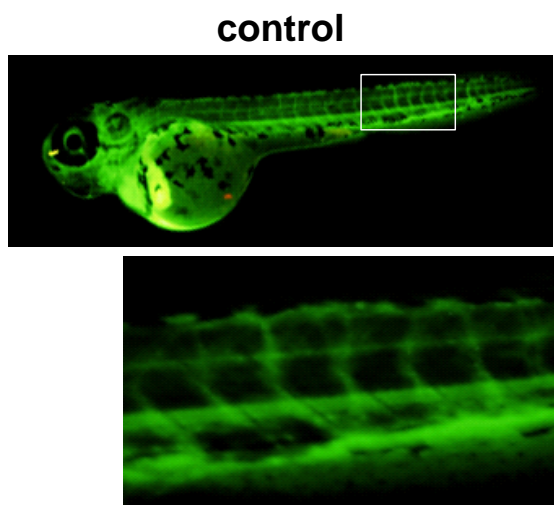

$+$
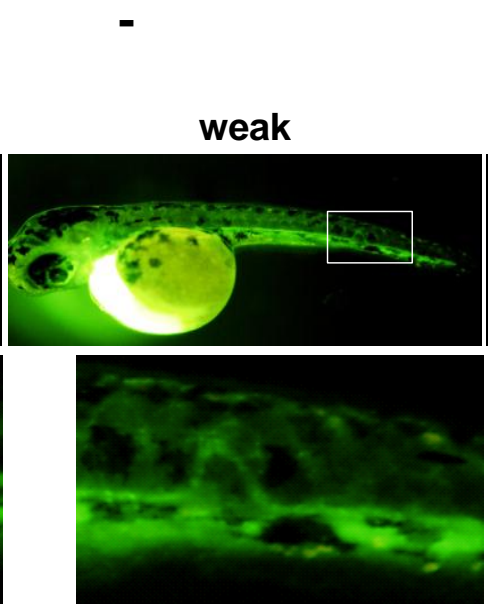

strong

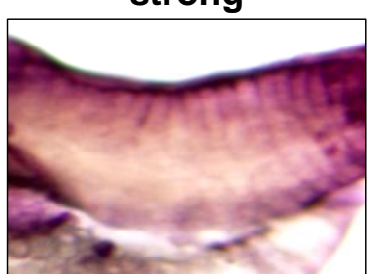

strong

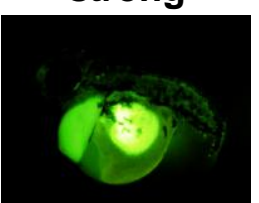

D

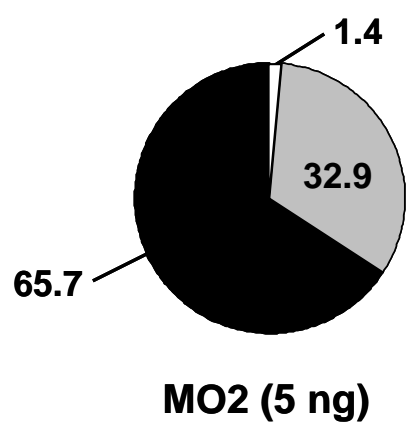

17.6

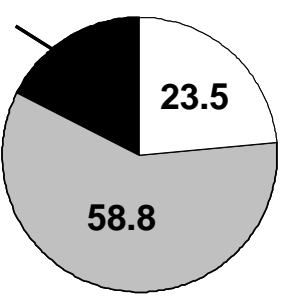

MO2 (2.5 ng)

$\square$ normal
$\square$ ISV defect
DA defect 
Fig. $5 \quad \mathrm{~A}$

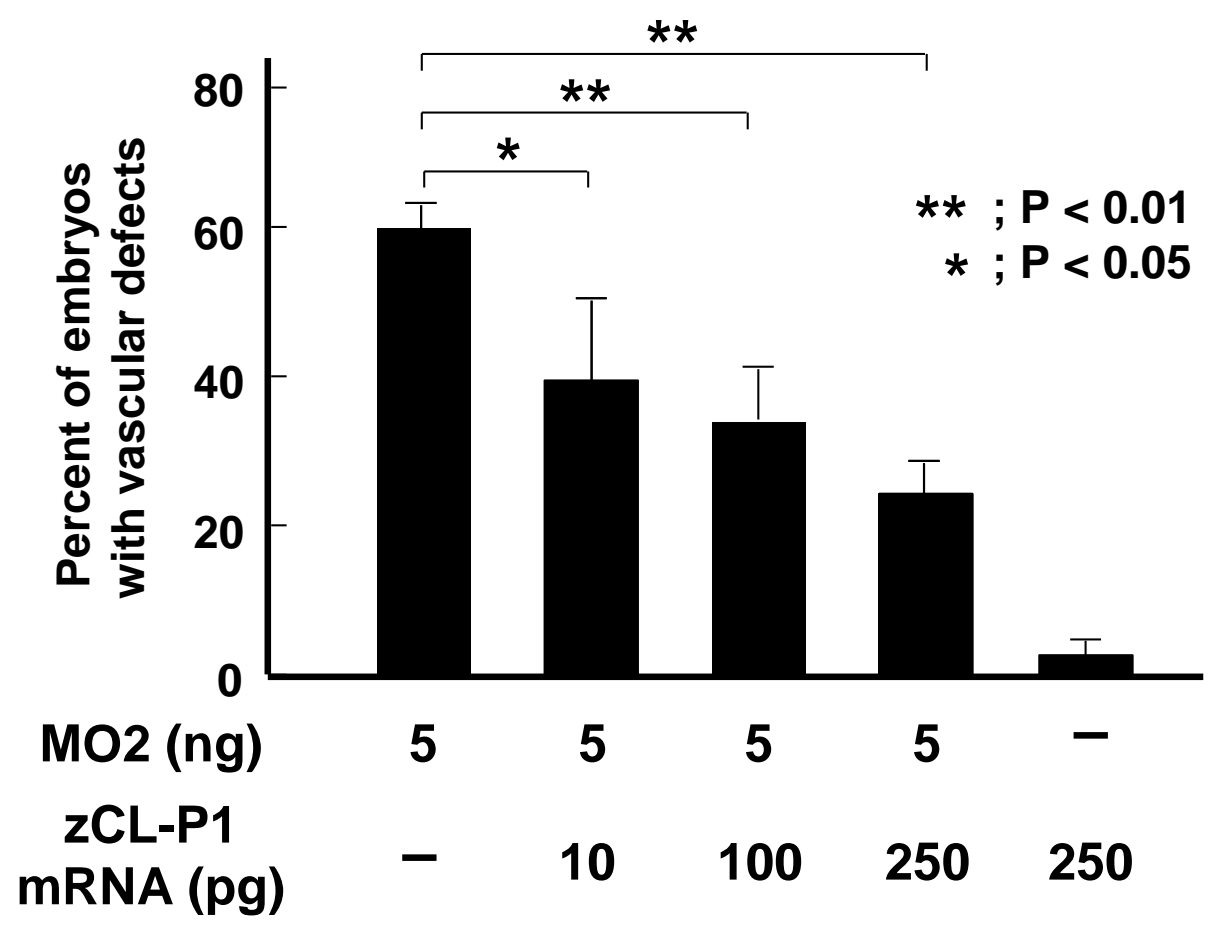

B

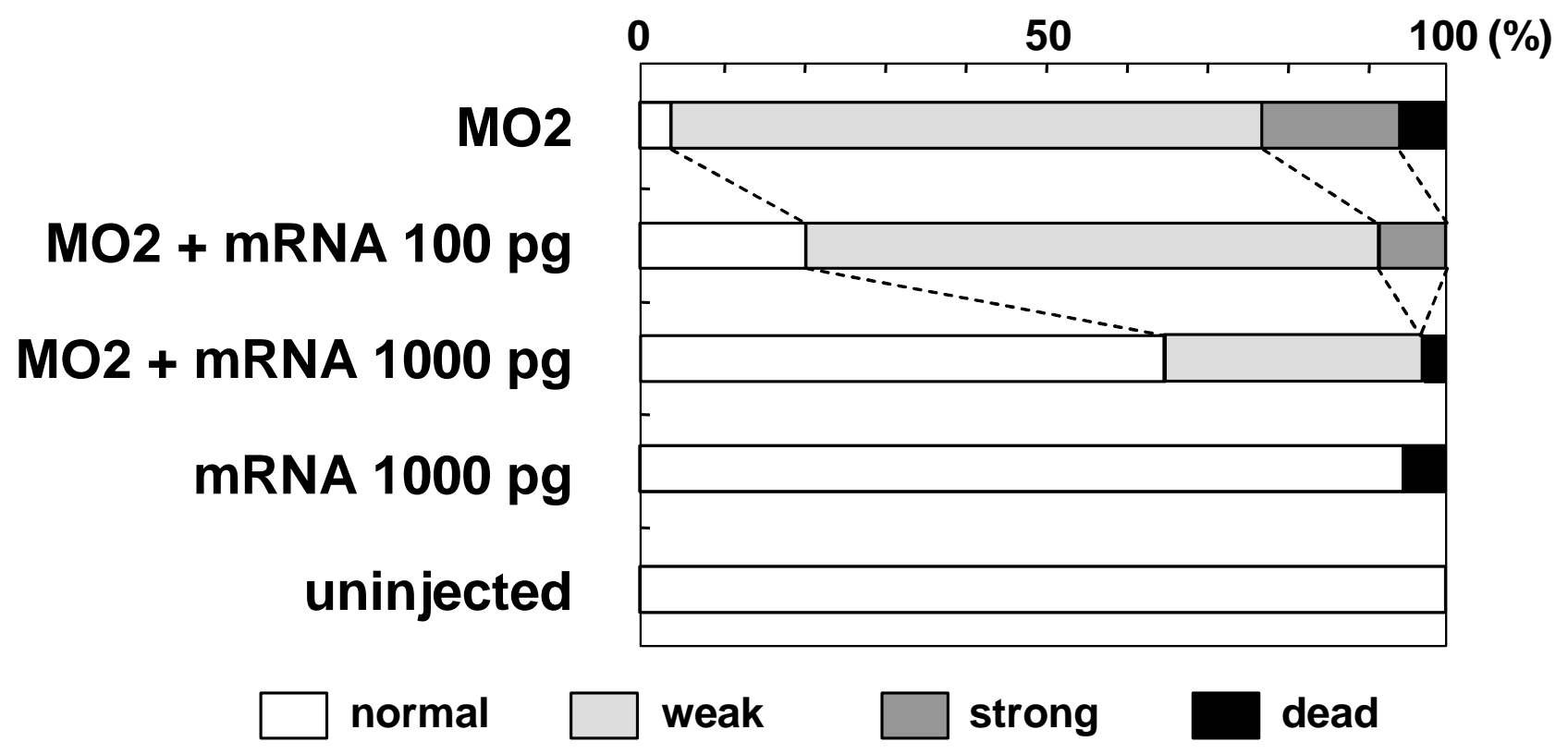


Fig. 6

A
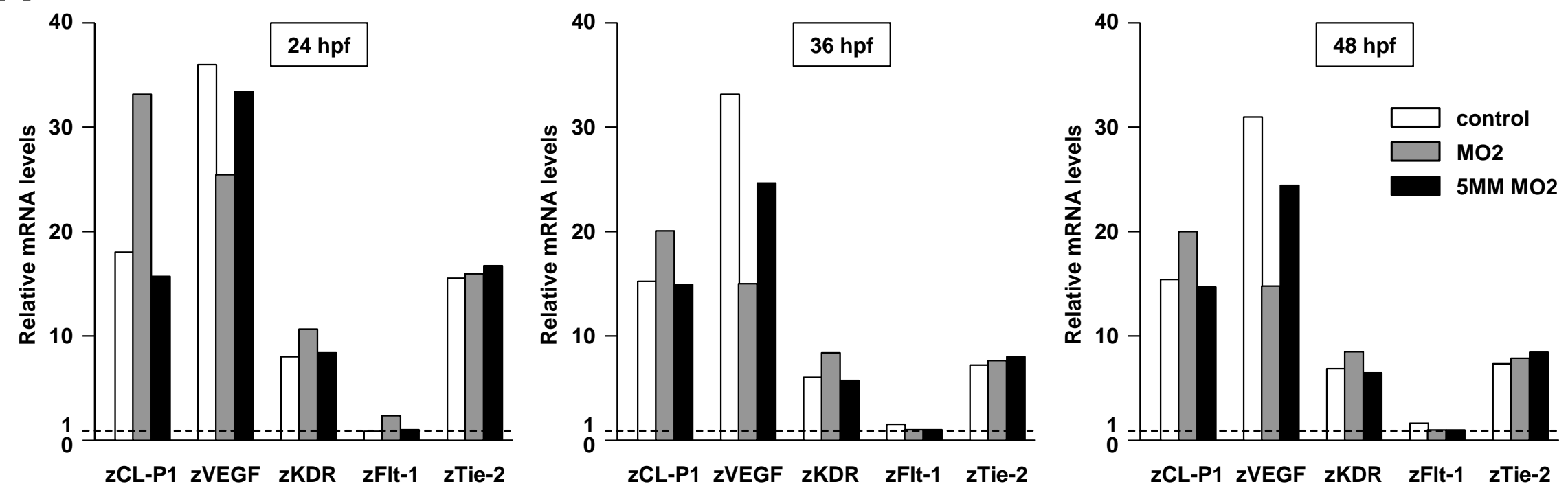

B

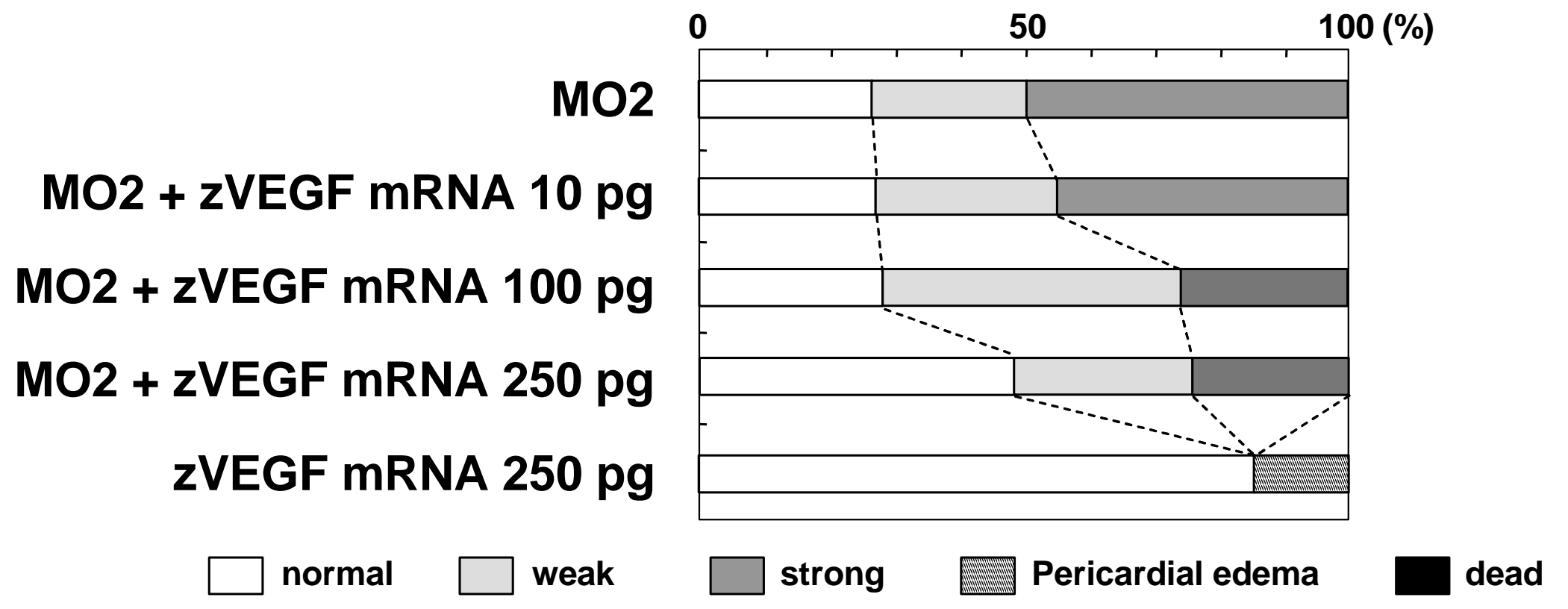


Table 1. Dose response effect of the antisense morpholino oligonucleotide MO2 on abnormal phenotype frequency in zebrafish embryos

\begin{tabular}{lccc} 
& & \multicolumn{2}{c}{ Abnormal phenotype frequency (\%) } \\
\cline { 3 - 4 } & Dose $(\mathrm{ng})$ & Experiment 1 & Experiment 2 \\
\hline control & 5 & 1.0 & 0 \\
MO2 & 1 & 3.6 & 0 \\
& 2.5 & 39.8 & 0 \\
& 5 & 1.0 & 0 \\
$5 \mathrm{MM}$ & 5 & & 03.3 \\
\hline
\end{tabular}

The design and sequences of the antisense morpholino oligonucleotide (MO2) and a five-basemismatched MO against MO2 are shown in Materials and methods. MOs were microinjected into 1-4 cell stage embryos, and phenotypes were observed at $48 \mathrm{hpf}$. Microscopic observation of embryos revealed that evident defects, such as shortness in body length and inadequate blood circulation, were frequently observed and classified as abnormal phenotypes. Each value represents the frequency (\%) of malformation in 52 - 118 zebrafish embryos. 
Table 2. Gene knockdown using MO2 caused the abnormal phenotype and death in zebrafish.

\begin{tabular}{|c|c|c|c|c|c|c|c|}
\hline & & & standard & MO2 (1ng) & MO2 (2.5ng) & MO2 (5ng) & uninjected \\
\hline total number & & & 52 & 76 & 60 & 60 & 160 \\
\hline normal (\%) & & & 48 (92.3) & 70 (92.1) & $53(88.3)$ & $15(25.0)$ & $155(96.9)$ \\
\hline \multirow{5}{*}{ phenotype (\%) } & \multirow{5}{*}{$48 \mathrm{hpf}$} & weak & 0 & $1(1.3)$ & $7(11.7)$ & $15(25.0)$ & 0 \\
\hline & & strong & 0 & 0 & 0 & $26(43.3)$ & 0 \\
\hline & & p. edema & 0 & 0 & $1(1.7)$ & $29(48.3)$ & 0 \\
\hline & & unknown & 0 & 0 & 0 & 0 & 0 \\
\hline & & total & 0 & $1(1.3)$ & $7(11.7)$ & $41(68.3)$ & 0 \\
\hline \multirow{6}{*}{ dead (\%) } & $24 \mathrm{hpf}$ & & $2(3.8)$ & $1(1.3)$ & 0 & $3(5.0)$ & $5(3.1)$ \\
\hline & \multirow{5}{*}{$48 \mathrm{hpf}$} & weak & 0 & $2(2.6)$ & 0 & 0 & 0 \\
\hline & & strong & 0 & 0 & 0 & 0 & 0 \\
\hline & & p. edema & 0 & 0 & 0 & 0 & 0 \\
\hline & & unknown & $2(3.8)$ & $2(2.6)$ & 0 & $1(1.7)$ & 0 \\
\hline & & total & $4(7.7)$ & $5(6.6)$ & 0 & $4(6.7)$ & $5(3.1)$ \\
\hline
\end{tabular}

Embryos at $48 \mathrm{hpf}$ that had received the standard oligonucleotide or $\mathrm{MO} 2(1,2.5,5 \mathrm{ng})$. The terms strong and weak indicate the level of abnormality in the gross appearance of embryos (Fig. 4B). The p. edema means the fish having pericardial edema. The rates of their respective phenotypes were MO2dose-dependent and oligonucleotide-sequence-specific. 